\title{
FORMACIÓN CÍVICA, ESTRUCTURA FUNDAMENTAL DE LA PARTICIPACIÓN CIUDADANA ${ }^{1}$
}

\section{Monterde Valenzuela María de los Ángeles ${ }^{2}$ Morales Tostado María del Carmen ${ }^{3}$}

SUMARIO: I. Introducción.II. Antecedentes históricos del proceso de formación cívica.III. Resultados obtenidos a partir de la encuesta. IV. Conclusiones y Propuestas

\section{RESUMEN}

México ha transitado por diferentes estrategias educativas, en las cuales la importancia de la formación cívica se ha reacomodado en la jerarquía. La cultura cívica forma parte de la formación ciudadana, se adquiere en el ámbito educativo; y se practica en el ámbito social. Tema importante de esa educación cívica es la democracia, concebida esta como una forma de gobierno en la que el poder político se ejerce por los ciudadanos y no solo por el grupo que ostenta el poder en un tiempo y espacio determinado.

En México el abstencionismo se presenta en el entorno electoral, como respuesta de ciudadanos a quienes no les interesa el quehacer de los políticos y del gobierno. Tampoco participan de forma activa en la solución de problemas que aquejan a su comunidad. Este suceso identifica la ausencia de formación cívica en los jóvenes mexicanos, y resalta la importancia de incluir en los procesos educativos de todos los niveles de educación pública a la educación cívica.

Las instituciones de educación superior tienen una misión educadora; son formadoras de ciudadanía, y sobre todo de personas íntegras que generen desarrollo en sus comunidades, fomenten la cohesión social, promuevan la formación de la identidad nacional y actúen con conciencia en la resolución de problemas contemporáneos.

Palabras clave : Educación cívica, desarrollo ciudadano y nacionalismo.

\footnotetext{
${ }^{1}$ Los resultados y propuestas de esta investigación se presentaron en en el "2020 Juried Virtual Conference" Western Social Science Association 62nd Annual Conference June 10 through September 30, 2020

${ }^{2}$ Docente investigadora de la Universidad Estatal de Sonora,correo electrónico angelesmonter50@hotmail.com

${ }^{3}$ Docente investigadora de Universidad de Sonora,correo electrónico, carmen.morales@unison.mx
} 


\title{
Formación cívica, estructura fundamental de la participación ciudadana
}

Volumen 12, Número 22 ene-jun 2020

Monterde Valenzuela María de los Ángeles y

Morales Tostado María del Carmen

\section{CIVIC TRAINING, FUNDAMENTAL STRUCTURE OF CITIZEN PARTICIPATION Monterde Valenzuela María de los Ángeles ${ }^{4}$ Morales Tostado María del Carmen ${ }^{5}$}

SUMMARY: I. Introduction. II. Historical background of the civic formation process III. Results obtained from the survey. IV. Conclusions and Proposals

\begin{abstract}
Mexico has gone through different educational strategies, in which the importance of civic education has been rearranged in the hierarchy. Civic culture is part of citizen training, it is acquired in the educational field; and it is practiced in the social sphere. An important theme of this civic education is democracy, conceived as a form of government in which political power is exercised by citizens and not only by the group that holds power in a given time and space.

In Mexico abstentionism occurs in the electoral environment, as a response from citizens who are not interested in the work of politicians and the government. Nor do they actively participate in solving problems that plague their community. This event identifies the absence of civic education in Mexican youth, and highlights the importance of including civic education in educational processes at all levels of public education.

Higher education institutions have an educational mission; they are educators of citizenship, and above all of upright people who generate development in their communities, promote social cohesion, promote the formation of national identity and act with conscience in solving contemporary problems.
\end{abstract}

Key words: Civic education, citizen development and nationalism.

\section{INTRODUCCIÓN}

Comprender la cultura como forma de vida de una comunidad humana, respecto a costumbres, creencias, aspiraciones, códigos de conducta, vestimenta, lenguaje, arte, cocina, ciencia, tecnología, religión, tradiciones e instituciones; hace reflexionar sobre la complejidad

\footnotetext{
${ }^{4}$ Research Professor, Sonora State University, México, e-mail: angelesmonter50@hotmail.com

${ }^{5}$ Research Professor, University of Sonora, México, e-mail: carmen.morales@unison.mx
} 
de entorno cultural en el que se está inmerso, y sobre la incidencia de estos factores en las decisiones.

Es por medio de la cultura que se promueve el desarrollo en general, y se establece una relación con otros objetivos como la democracia, la participación ciudadana, la conservación del medio ambiente, la preservación de los valores comunitarios y la protección de las instituciones civiles.

Por estas razones, para hablar de cultura cívica, democracia y participación ciudadana, es necesario abordar el tema desde diversas perspectivas; considerando su transcendental función en los procesos educativos formales que tienen el compromiso de formar ciudadanos libres, conocedores de sus derechos y obligaciones; pero sobre todo comprometidos con su entorno social.

La formación ciudadana involucra diversos factores y requiere de un largo tiempo para observar sus efectos; inicia a temprana edad, en el hogar y se escala durante la niñez y juventud. Su construcción es intencional, estratégicamente planeada, estructurada, y fortalecida con instituciones que forman su cadena de valor desde la familia-escuela-sociedad-gobierno y nuevamente sociedad. Con ello, se edifica al ciudadano que conoce y vive las virtudes cívicas como la tolerancia, el respeto a los derechos humanos, la transparencia, el trabajo, el patriotismo y la paz.

Para tener un referente de lo que opinan los estudiantes universitarios sobre el tema de Formación Ciudadana, se inició la presente investigación y se diseñó una encuesta en la que se incluyen 10 preguntas y con sus respuestas permite conocer las opiniones en temática relacionada con los aspectos fundamentales de análisis de este trabajo. En ella se develan interesantes datos; como que un $90.3 \%$ de los estudiantes encuestados conocen los asuntos del entorno político por redes sociales. Igualmente expresan que los problemas de la sociedad deben ser resueltos con la participación de la sociedad y gobierno; además al cuestionar si considera que México es un país democrático un 69.9 \% responde afirmativamente "que en parte".

Con los datos obtenidos, puede deducirse que la educación en México requiere de un impulso sostenido, progresivo y práctico en el tema cultura cívica; en especial sobre el entendimiento e incorporación práctica de valores como democracia, participación, 


\section{Formación cívica, estructura fundamental de la participación ciudadana}

Volumen 12, Número 22 ene-jun 2020

Monterde Valenzuela María de los Ángeles y

Morales Tostado María del Carmen

transparencia y patriotismo, entre otros, para que se puedan observar resultados en la formación de las generaciones futuras de ciudadanos.

\section{Objetivo General}

Analizar los antecedentes y evolución de los aspectos principales que participan en la cultura cívica de los universitarios, su percepción sobre democracia y participación ciudadana en los procesos electorales, a fin de determinar las áreas de oportunidad existentes en la formación universitaria, y en base a ellas incorporar contenidos cívicos que mejoren las mallas curriculares 2020.

\section{Hipótesis}

La cultura cívica de nuestros estudiantes proviene de fuentes institucionales como la familia o la escuela, y los convierte en ciudadanos informados, responsables que participan activamente en la sociedad y que perciben la democracia como factor indispensable para dar legitimidad a un gobierno.

\section{ANTECEDENTES HISTÓRICOS DEL PROCESO DE FORMACIÓN CÍVICA EN MÉXICO.}

En la historia de México, se han establecido como competencias del Estado la formación o instrucción cívica y política. Dentro de los procesos históricos es importante mencionar lo plasmado en la Constitución Política de los Estados Unidos Mexicanos (CPEUM), de 1857 donde se suprimían los beneficios del clero y del ejército y donde se establecía la igualdad de todos los ciudadanos ante la Ley. En 1874, se describió los ámbitos de competencia de la Iglesia y el Estado, quedando descrito que la educación debe ser laica.

A finales del siglo XIX, en el período correspondiente al Porfiriato, la educación cívica se circunscribía a fomentar valores patrios hacia el país y para ello se crearon símbolos y héroes que propiciaban la cohesión social. Dentro de los valores que se fomentaban en esta época figuraban la libertad, orden, obediencia, respeto, puntualidad, amor filial, gratitud y amor a los demás, entre otros.

En ese periodo se realizaron cambios importantes en el ámbito educativo que se pueden agrupar en tres aspectos: la educación laica, la educación obligatoria y la educación gratuita. $Y$ se puso en marcha una campaña de alfabetización en todo el país, que contribuyó de manera significativa a la construcción de un mejor país. 
Posteriormente en la época de la Revolución Mexicana, el interés de los ciudadanos se concentró en la lucha por la justicia social, en el ámbito educativo se identificaba deficiencias por falta de una adecuada organización y planeación del quehacer educativo, tan necesario para la formación integral de los niños y jóvenes. Lo que dio lugar a cambios trascendentales, uno de ellos es la creación en 1921 de la Secretaría de Educación Pública, siendo publicado en el Diario Oficial de la Federación el 3 de octubre de 1921 y el 12 de octubre del mismo año, asume la titularidad de la SEP el Lic. José Vasconcelos Calderón.

Un factor trascendental que distinguió a esta época fue la entrega de los libros de texto gratuitos en 1959, lo que permitió conocer y uniformar los contenidos de la enseñanza que se proporcionaba a la población infantil mexicana.

Más adelante, se impulsó una reforma que dio preferencia a la esfera cognoscitiva, quedando la educación cívica como una materia teórica desligada de la práctica. Todo ello, relegó la formación ciudadana y al paso de los años se concibió al ciudadano como un simple depositario de derechos al que no se le fomentaba su participación en la vida política de su país.

En el período de 1970 a 1976, sucede otro cambio importante en el entorno educativo y se relaciona con el plan de estudios de educación básica que se pretendía pudiera procurar una formación armoniosa, que incluyera la capacidad creadora, educación democrática, conciencia histórica, entre otras. Fue entonces, que se forman las áreas de conocimiento, y corresponde a las ciencias sociales incorporar los temas que nos ocupan: economía, historia, geografía y política.

En ese tiempo, ya se realizaban investigaciones y algunas voces se alzaron para dar a conocer las críticas al esquema curricular por áreas de conocimiento. Uno de ellos es Guevara (2016:77), quien señala lo siguiente:

... la formación escolar se ve condicionada por tres factores: ausencia de una preocupación explícita por la formación de la personalidad moral del alumno, un sistema educativo centralista y burocrático en el que imperó una concepción jerárquica de la relación maestro alumno y una gestión institucional vertical. ${ }^{6}$

\footnotetext{
${ }^{6}$ Vide: Guevara Niebla,G., Democracia y Educación en Cuaderno de Divulgación de la Cultura Democrática \#16,México,INE,2016, p.77[en línea] Disponible en: https://portalanterior.ine.mx/archivos2/portal/historico/contenido/recursos/IFE-v2/DECEYEC/DECEYECCuadernosdeDivulgacion/docs/16.pdf
} 


\section{Formación cívica, estructura fundamental de la participación ciudadana}

Volumen 12, Número 22 ene-jun 2020

Monterde Valenzuela María de los Ángeles y

Morales Tostado María del Carmen

Su voz no consigue atraer la atención de las personas que toman las decisiones sobre las políticas públicas educativas y no hay cambios en ese modelo educativo.

En la década de los ochenta, la sociedad mexicana presenta una serie de situaciones que hacen que se revisen las condiciones sociales del país ${ }^{7}$ buscando respuesta a los resultados obtenidos en el entorno político electoral, priorizando la preocupación gubernamental del momento político (abstencionismo electoral y desprestigio de los servidores públicos) y discriminando la formación moral ciudadana de los jóvenes.

La televisión, fue el medio de pasatiempo más utilizado en esta década, y contribuyó notablemente en la adquisición de nuevos hábitos de consumo, utilización del tiempo, cambios en las dinámicas familiares y formación de la conciencia individual y colectiva.

El revisar estos fenómenos sociales, obligó a considerar importante la educación cívica que promueve la formación de los ciudadanos y adquiere un renovado interés y se retoma nuevamente el civismo dando énfasis a la educación para la regulación del propio comportamiento y respuesta a estímulos sociales y políticos y formación para la convivencia social.

Todo ello, motivó una reforma educativa que da lugar al ordenamiento curricular por materias, apareciendo como asignatura la educación cívica. Así mismo, se elaboran nuevos libros de texto para que correspondan a los cambios curriculares. En dichos libros se incluyen temática sobre los derechos humanos.

Los medios masivos de comunicación en su momento, y ahora el internet y los dispositivos móviles, han influido en la formación de perspectiva que tienen los jóvenes mexicanos, la adquisición de valores diferentes a los de su cultura, la nueva forma de interactuar en el mundo; ha re-definido su cultura, su canal de aprendizaje, su familia, sus intereses y conceptos.

El tema de los valores recobra importancia y se plasma en varios documentos, en el Programa Nacional de Educación 2001-2006 se consigna que la educación debe ser equitativa, de calidad, pertinente, incluyente y debe formar de manera integral con la construcción de una ética pública.

\footnotetext{
7 Vide:Sesento García, L., La influencia de los medios de comunicación en las contribuciones a las ciencias sociales, Eumed,2015,p.s/n
} 
Posteriormente, conforme al Plan Nacional de Educación en Derechos Humanos (PlanEDH:20062010), se traza la política educativa desde el enfoque de los derechos humanos y la participación ciudadana, con base en nuestros ordenamientos nacionales y los mecanismos internacionales en la materia y comprende objetivos, propuestas, solicitudes específicas a las autoridades educativas, recomendaciones a la sociedad civil, estrategias, así como campos temáticos de intervención y líneas de acción,de la política educativa vuelta de nuevo a capacitar a los ciudadanos del hoy y del mañana y fomentar en ellos su intervención activa en los asuntos de interés público. ${ }^{8}$

En un estudio comparado de 120 planes de estudio universitarios (Guerrero Useda \& Gómez Paternina, 2013), develan que la educación moral de la persona permanece ausente del currículo universitario. La enseñanza de la ética ha ganado espacio de manera selectiva en algunas mallas curriculares de programas educativos universitarios particulares, quedando pendiente su desarrollo didáctico. Aproximadamente en la mitad de los programas comparados en este estudio, se incluye en el plan de estudios al menos un curso asociado a la ética, predominando el sentido normativo o de carácter informativo.

Con todo lo señalado anteriormente, se puede identificar que en los planes de estudios de universidades privadas hay mayor interés en incluir la temática de educación cívica. En las universidades públicas el proceso de adicionar dicha temática ha sido más lento.

Como corolario, se comparte la aportación de la Organización de los Estados Americanos (OEA) sobre este tema formativo: la Carta Democrática Interamericana (2001), en ella se plasma el siguiente:

Considerando que la educación es un medio eficaz para fomentar la conciencia de los ciudadanos con respecto a sus propios países y, de esa forma, lograr una participación significativa en el proceso de toma de decisiones, y reafirmando la importancia del desarrollo de los recursos humanos para lograr un sistema democrático y sólido.

Por lo planteado anteriormente, se puede deducir que la educación para la democracia es sumamente importante para los países. En virtud, de que todos aspiran a tener ciudadanos con formación de valores cívicos y éticos, informados y críticos de su entorno, participativos en la solución de los problemas sociales.

\footnotetext{
8 Ramírez,Gloria, Plan Nacional de educación en Derechos humanos, Primera edición,México 2006,Cátedra UNESCO de Derechos Humanos de la UNAM Facultad de Ciencias Políticas y Sociales (FCPS)
} 


\section{Formación cívica, estructura fundamental de la participación ciudadana}

Volumen 12, Número 22 ene-jun 2020

Monterde Valenzuela María de los Ángeles y

Morales Tostado María del Carmen

Ha sido largo el camino para delinear las políticas educativas en el tema de cultura cívica, como se pudo apreciar en los párrafos anteriores se cambia constantemente de estrategias según la teoría o modelo con el cual se identifiquen los servidores públicos que tienen la facultad de tomar decisiones en este tema. Sin embargo, aunque se ha logrado mediante investigaciones educativas obtener información que permite decidir y diseñar las acciones que nos llevarán a formar de manera integral a los ciudadanos y con ello consolidar el proyecto de nación que todos queremos, seguimos con áreas de oportunidad en el tema de cultura cívica, en virtud de que es un proceso dinámico que se vive no solo en la escuela, sino que involucra a toda la sociedad y en todas las etapas de formación de una persona.

Al abordar el tema de democracia, se parte de las definiciones que sobre esa palabra emite la Real Academia Española:

1. Forma de gobierno en la que el poder político es ejercido por los ciudadanos.

2. Doctrina política según la cual la soberanía reside en el pueblo, que ejerce el poder directamente o por medio de representantes.

Al reflexionar sobre las definiciones, se confirma que la soberanía reside en el pueblo y hasta ahora se gobierna para el pueblo y es necesario cambiar el paradigma y ahora gobernar con el pueblo.

En ese tenor, la Organización de los Estados Americanos (OEA) señala en la Carta Democrática los valores y principios que se requieren en la democracia: libertad, igualdad y justicia social que son intrínsecos a la democracia. Promover y proteger los derechos humanos es condición fundamental para la existencia de una sociedad democrática, y reconociendo la importancia que tiene el continuo desarrollo y fortalecimiento del sistema interamericano de derechos humanos para la consolidación de la democracia.

REAFIRMANDO que el carácter participativo de la democracia en nuestros países en los diferentes ámbitos de la actividad pública contribuye a la consolidación de los valores democráticos y a la libertad y la solidaridad en el Hemisferio.

Estos puntos señalados desde el año 2003 en la Carta Democrática de la OEA siguen teniendo relevancia en el sentido de que no se ha podido lograr una democracia responsable.

En virtud de que, para comprender la formación y participación ciudadana, es necesario analizar el significado que tiene participar y para ello, Mauricio Merino expresa lo siguiente: 
PARTICIPAR, en principio, significa "tomar parte": convertirse uno mismo en parte de una organización que reúne a más de una sola persona. Pero también significa "compartir" algo con alguien o, por lo menos, hacer saber a otros alguna noticia. De modo que la participación es siempre un acto social: nadie puede participar de manera exclusiva, privada, para sí mismo. ${ }^{9}$

Para analizar con mayor detalle el tema, es importante compartir lo que plantea Conde que expresa lo siguiente:

Se presenta a la formación ciudadana como una educación en valores porque el ciudadano democrático es un sujeto cuya cualidad moral se expresa en su compromiso con el bienestar común, en la capacidad de convertir los principios y valores de la democracia en criterios de juicio y acción, reconocedor del otro, con alto sentido de la justicia, la legalidad y de sus responsabilidades humanas. Pero también es una educación en derechos humanos, porque el ciudadano se constituye en el ejercicio de éstos. ${ }^{10}$

Por tanto, para construir un mejor país, es necesario llevar a la práctica las acciones que promuevan la participación ciudadana y fomentar que cada mexicano se convierta en agente de cambio y entre todos difundir los derechos y obligaciones que tienen los ciudadanos.

Al concientizar a los ciudadanos sobre lo importante de su participación, se podrá influenciar en la toma de decisiones, en la formulación, instrumentación, monitoreo y evaluación de políticas públicas sobre la acción gubernamental.

En México están emergiendo nuevas formas de participación pública, se han abierto nuevas fórmulas de participación ciudadana en la elaboración de políticas públicas en todo el conjunto de la democracia representativa. Los ciudadanos demandan a sus gobiernos la oportunidad de participar activamente en la elaboración de las políticas públicas que conducen el destino del país.

La Organización para la Cooperación y Desarrollo Económico, elabora y presenta el documento Gobierno Abierto en América Latina, señalando que la mayoría de los países

\footnotetext{
9 Vide: Merino, M., La participación Ciudadana en la Democracia en Cuaderno de divulgación de la Cultura Democrática \#4.México,INE,2016,p.12 [en linea]Disponible en:

https://portalanterior.ine.mx/archivos2/portal/historico/contenido/recursos/IFE-v2/DECEYEC/DECEYECCuadernosdeDivulgacion/docs/04.pdf

${ }^{10}$ Vide:Conde, S., Formación Ciudadana en México, en Cuadernos de Divulgación de la Cultura Democrática \#32,méxico, INE,2016,p. 16 [en línea]Disponible en: https://portalanterior.ine.mx/archivos2/portal/historico/contenido/recursos/IFE-v2/DECEYEC/DECEYECCuadernosdeDivulgacion/docs/32.pdf
} 


\section{Formación cívica, estructura fundamental de la participación ciudadana}

Volumen 12, Número 22 ene-jun 2020

Monterde Valenzuela María de los Ángeles y

Morales Tostado María del Carmen

miembros han evolucionado hacia un gobierno más abierto y transparente (OCDE, 2015), donde se permite la participación ciudadana en la toma de decisiones ${ }^{11}$.

Sin embargo, se debe ser considerar que la participación por sí sola no garantiza que haya democracia o que mejore el desempeño del gobierno, en ese sentido Merino afirma que la participación no es suficiente para entender la dinámica de la democracia; pero sin participación, sencillamente la democracia no existiría. Esta propuesta deja claro que la democracia requiere siempre de la participación ciudadana: con el voto y más allá de los votos. ${ }^{12}$

Reiterando lo anterior, y continuando con el sentido constructivo de la democracia, Sojo, expresa que para ser sólida, una democracia necesita mucho más que elecciones libres y equitativas; afirmando que, en una sociedad plural como la mexicana, la convivencia diaria tiene que fundamentarse en virtudes cívicas como la tolerancia, el respeto a las leyes y a los derechos de terceros y, de manera específica a los derechos de las minorías. ${ }^{13}$

En México a partir del año 2000 se han establecido condiciones que fomentan las organizaciones civiles, se puede mencionar la creación de un marco normativo para identificar y regular la actividad de las organizaciones civiles, el cual sienta las bases para la institucionalización de la relación con las organizaciones civiles. Adicionalmente se estableció el registro nacional de instituciones y organizaciones políticas sociales y civiles, para identificar aquellas con causas similares, a fin de propiciar su trabajo conjunto.

En ese mismo sentido, la formación integral que incluye la formación moral, tiene como propósito educar a las nuevas generaciones con los principios de igualdad y justicia. En este sentido, es importante para este tema lo que señala Pablo Latapí

... junto a las finalidades políticas de educar a las nuevas generaciones en conformidad con los principios republicanos, figuraba el propósito de impartirles una formación moral que diera solidez y consistencia al proyecto político nacional ${ }^{14}$

\footnotetext{
${ }^{11}$ OCDE. (2015). Gobierno Abierto en América Latina. Estudios sobre Gobernanza Pública, España, OCDE Publishing,2015,p.s/n [ en línea] Disponible en: http://www.oecd.org/gov/gobierno-abierto-en-america-latina9789264225787-es.htm

12 Merino, op.cit.,p.19

13 Vide:Sojo-Garza Aldape, E.,De la alternancia al desarrollo,en Políticas públicas del gobierno del cambio. México: Fondo de Cultura Económica,2005,p.s/n

${ }^{14}$ Latapí Sarre, P., El Debate sobre los valores en la Escuela Mexican,México, Fondo de Cultura Económica,2013,p13
} 
Como se puede apreciar, están fuertemente vinculados los procesos educativos, los procesos políticos y la formación cívica.

La formación ciudadana involucra muchos factores y requiere de un largo período de tiempo, en el cual se llevan a cabo procesos educativos formales e informales, en ambos procesos se busca transmitir conocimientos, habilidades y valores que guíen la conducta de los seres humanos y los conviertan en buenos ciudadanos.

La Ley General de Transparencia y Acceso a la Información Pública (LGTAIP), publicada en el Diario oficial de la federación mexicana el 4 de mayo de 2015, es parte de un proyecto diseñado para la formación de los ciudadanos, donde se busca incorporar los valores de responsabilidad, respeto, honestidad, tolerancia, entre otros; dicho proyecto es de largo plazo y debe darse desde los espacios formativos, tales como escuelas y universidades y organizaciones de la sociedad civil.

Para visualizar el escenario futuro de formación de ciudadanía, es necesario compartir las seis etapas del desarrollo ciudadano que integra todo el proceso de vida de una persona. En el cuadro 1 se presenta esa información:

Cuadro 1. Las seis etapas del desarrollo ciudadano

Etapa $\quad$ Descripción




\section{Formación cívica, estructura fundamental de la participación ciudadana}

Volumen 12, Número 22 ene-jun 2020

Monterde Valenzuela María de los Ángeles y

Morales Tostado María del Carmen

\begin{tabular}{|c|c|}
\hline 1 & $\begin{array}{l}\text { Ciudadanía biológica: dependencia infantil y crianza materna. Del } \\
\text { nacimiento a los } 3 \text { años. }\end{array}$ \\
\hline 2 & $\begin{array}{l}\text { Ciudadanía familiar: ampliación de las influencias familiares. De los } 4 \text { a } \\
\text { los } 5 \text { años. }\end{array}$ \\
\hline 3 & $\begin{array}{l}\text { Ciudadanía social formativa: iniciación de las dependencias no } \\
\text { biológicas. De los } 6 \text { a los } 9 \text { años. }\end{array}$ \\
\hline 4 & $\begin{array}{l}\text { Ciudadanía social estratificada: desarrollo de categorías sociales y } \\
\text { conductas de pertenencia a grupos. De los } 10 \text { a los } 12 \text { años. }\end{array}$ \\
\hline 5 & $\begin{array}{l}\text { Ciudadanía de grupo por edad cronológica: relaciones sociales } \\
\text { horizontales, dentro y entre grupos. De los } 13 \text { a los } 15 \text { años. }\end{array}$ \\
\hline 6 & $\begin{array}{l}\text { anía social compleja: búsqueda de ubicación y de la identidad } \\
\text { De los } 17 \text { a la edad adulta. }\end{array}$ \\
\hline
\end{tabular}

Fuente: Elaboración propia a partir de Guevara (2016: 96) quien señala como fuente a T.L. Dyneson y R. E. Gross, "An Ecclectic Approach to Citizenship: Development Stages", en Social Studies, vol. 76, enero-febrero de 1985, pp. 23-27.

El Gobierno de México en 2019 en su Plan Nacional de Desarrollo, establece algunos ejes temáticos en los que incorpora temas como democracia y la participación ciudadana; y en sus políticas de gobierno establece el garantizar empleo, educación, salud y bienestar. Describe enfáticamente que Democracia significa "el poder del pueblo" y expresa vehementemente su estrategia de conseguir en México una democracia participativa para socializar el poder político e involucrar a la sociedad en las grandes decisiones nacionales. Para ello, establece la consulta popular o ciudadana, la revocación de mandato y las asambleas comunitarias como instancias efectivas de participación.

El Plan Nacional de Desarrollo 2018-2024, está lejano a ser un plan estratégico porque carece de un análisis profundo de la situación del país, objetivos y estrategias; y se considera más bien un texto teórico que cumple con el mandato establecido en el artículo 26 Constitucional, el cual abunda en adjetivos calificativos, pero no señala acciones concretas; contiene cuartillas del pasado, pero poca información objetiva sobre la situación real del país. ${ }^{15}$

\footnotetext{
15 González, A. Plan-nacional-de-desarrollo-2008-2014.México, 2019 [en línea]Disponibe en: https://newsweekespanol.com/2019/05/opinion-plan-nacional-de-desarrollo-fin-del-oscurantismo-neoliberal/
} 


\section{Metodología}

La presente investigación es de tipo descriptivo, para su realización se han consultado libros, artículos en revistas especializadas, documentos y sitios web oficiales de instituciones públicas.

Los resultados de esta investigación consideran, además, una encuesta en línea que se diseñó en la plataforma Google Formularios; y cuyas respuestas expresan la opinión de 113 estudiantes universitarios.

Los estudiantes se convocaron de forma aleatoria y se les invitó a participar dando su opinión sobre el tema cultura cívica democrática.

\section{Preguntas de investigación:}

1. ¿Cómo se percibe la cultura cívica considerando los medios de información que tiene un ciudadano y la influencia de los grupos que participan en la toma de decisiones?

2. ¿Considera satisfactoria la democracia en México?

3. ¿Considera que México cuenta con una ciudadanía participativa y comprometida con el buen desempeño de los asuntos públicos de su comunidad?

4. ¿Qué tanto cree usted que los ciudadanos pueden influir en las decisiones de un gobierno?

RESULTADOS OBTENIDOS A PARTIR DE LA ENCUESTA A ESTUDIANTES UNIVERSITARIOS.

De la aplicación de encuesta a 113 estudiantes universitarios, se obtienen los siguientes resultados:

En el cuadro 2, se muestra que el $63.7 \%$ de los participantes en el estudio son de género femenino, y el $36.3 \%$ son de género masculino. La edad de los estudiantes universitarios participantes en la encuesta, indica que el $85 \%$ pertenece al rango de $18-24$; un $10.6 \%$ al rango de $25-34$ años, un $2-7 \%$ al rango de $35-44$ años y el $1.8 \%$ corresponde a alumnos mayores de 50 años.

Cuadro 2. Datos generales de la población universitaria objeto de estudio. 


\section{Formación cívica, estructura fundamental de la participación ciudadana}

Volumen 12, Número 22 ene-jun 2020

\begin{tabular}{|c|c|c|}
\hline \multicolumn{3}{|c|}{$\begin{array}{c}\text { Datos Generales de la Población } \\
\text { Universitaria UNISON-UES }\end{array}$} \\
\hline \multirow[t]{2}{*}{ Género } & Mujer & $63.70 \%$ \\
\hline & Hombre & $36.30 \%$ \\
\hline \multicolumn{3}{|l|}{ Edad } \\
\hline 18-24 & \multicolumn{2}{|r|}{$85.00 \%$} \\
\hline $25-34$ & \multicolumn{2}{|r|}{$10.60 \%$} \\
\hline $35-44$ & \multicolumn{2}{|r|}{$2.70 \%$} \\
\hline Iayor de 50 & \multicolumn{2}{|r|}{$1.80 \%$} \\
\hline
\end{tabular}

Medio más utilizado para informarse del entorno político

\begin{tabular}{|r|r|}
\hline Radio & $16.80 \%$ \\
\hline Televisión & $\mathbf{2 3 . 0 0 \%}$ \\
\hline Periódicos-Revistas & $10.60 \%$ \\
\hline Internet-Redes Sociales & $\mathbf{9 0 . 3 0 \%}$ \\
\hline Rumores & $10.60 \%$ \\
\hline No me interesa & $0.90 \%$ \\
\hline No contestó & $0.90 \%$ \\
\hline
\end{tabular}

Monterde Valenzuela María de los Ángeles y

Morales Tostado María del Carmen

Fuente: Elaboración propia con información de la encuesta.

En relación a la cuestión de cuál es el medio de información más utilizado para conocer asuntos del entorno político, resulta que el $90.3 \%$ utiliza redes sociales, seguido de un $23 \%$ que se informa por la televisión, y un $16.8 \%$ por medio de la radio. En igualdad de proporciones, un $10.6 \%$ de los estudiantes se informa por periódicos y revistas; así como por comentarios o rumores. Las opciones de "no me interesa" o "no contestó" tuvieron la mínima respuesta de $0.9 \%$, resultado que afirma que los jóvenes universitarios se interesan por conocer asuntos políticos y en su mayoría lo hacen por redes sociales.

Al referirse a la influencia que tienen algunos actores en el entorno democrático, para la toma de decisiones sobre asuntos que impactan en la sociedad, los estudiantes opinaron que los partidos políticos influyen mucho, seguido del presidente y luego de las grandes empresas. En cuarto orden de importancia los ciudadanos, seguido de los sindicatos y por último de las agrupaciones ciudadanas.

Un dato relevante fue la opinión sobre responsabilidad en problemas de la sociedad, en la que los estudiantes plasman acuerdo casi unánime al afirmar "Los problemas de la sociedad, deben ser resueltos con la participación de la sociedad y del gobierno"

En referencia con la hipótesis planteada en esta investigación, en sentido de suponer que el país tiene una cultura cívica que permite la formación de una ciudadanía informada; 
responsable que participa activamente, y que percibe la democracia como factor indispensable para legitimar a un gobierno. La gráfica 1 muestra que el lugar principal donde se reflexiona sobre temas políticos es en la escuela, con un $31.9 \%$ de las menciones, la familia con un $23 \%$ y los amigos con un $13.3 \%$ de las participaciones de los estudiantes universitarios. Un $12.4 \%$ manifiesta que no le interesa la política y evita el tema. Interesante dato a contrastar con estas respuestas, resulta saber que las fuentes de información sobre el entorno político más utilizadas son las redes sociales con un $90.30 \%$ y la televisión con un $23 \%$; lo cual supone una fuente de información no muy confiable o poco formativa.

Gráfica 1. Lugar donde se reflexiona sobre temas políticos en México.
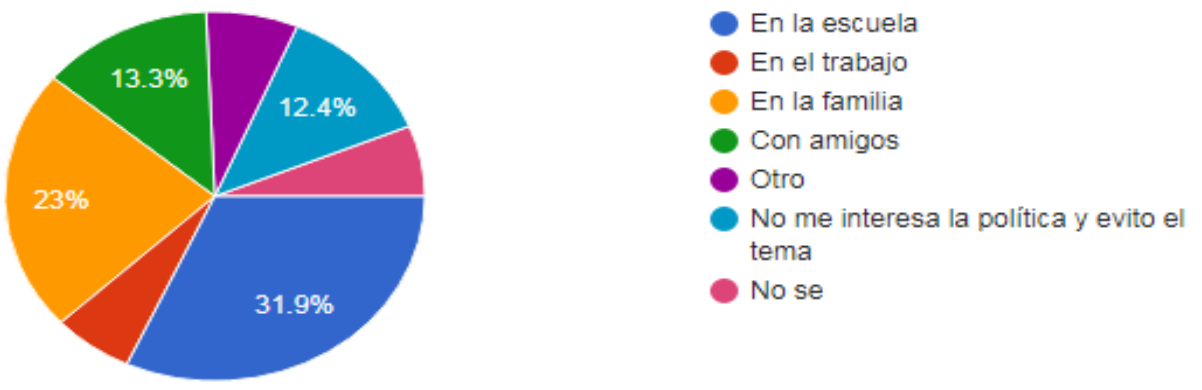

Fuente: Elaboración propia con información de la encuesta.

Relevante respuesta se muestra en el gráfico 2, ante la cuestión de si considera que México es un país democrático; a lo que el $69.9 \%$ de los estudiantes universitarios perciben que "en parte", mientras que un $17.7 \%$ afirma que "No es un país democrático"; y sólo un $7.1 \%$ afirma que "Si es un país democrático"

Gráfico 2. Percepción de la democracia en México, según los estudiantes universitarios objeto de estudio.

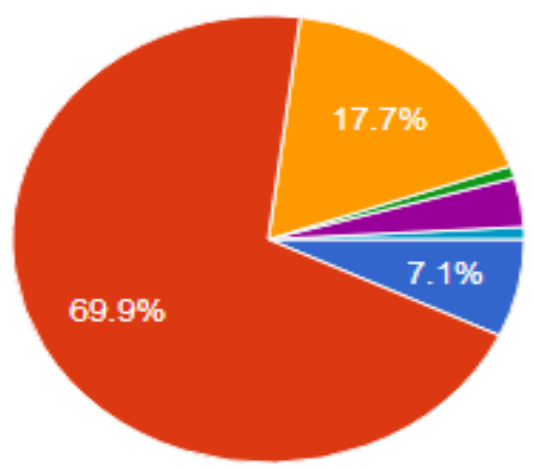

Fuente: Elaboración propia con información de la encuesta. 


\section{Formación cívica, estructura fundamental de la participación ciudadana}

Volumen 12, Número 22 ene-jun 2020

Monterde Valenzuela María de los Ángeles y Morales Tostado María del Carmen

La proporción de estudiantes universitarios que está satisfecho con la democracia de México (Gráfico 3), medido en escala del 1 al 5; siendo 1 muy satisfecho y 5 nada satisfecho, corresponde al $52.2 \%$ de estudiantes ubicados en el punto medio (3) de la escala, y en igualdad de porción 19.5\% están 2 grupos (4 y 5 de la escala) en el extremo de "nada satisfecho".

Gráfico 3. Escala de satisfacción con la democracia de México.

60

40

20

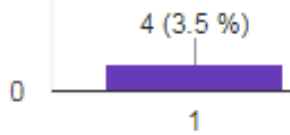

$6(5.3 \%)$

2

\section{$59(52.2 \%)$}

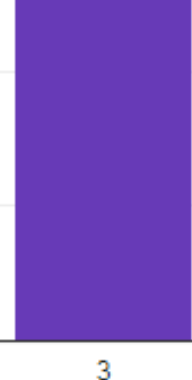

3

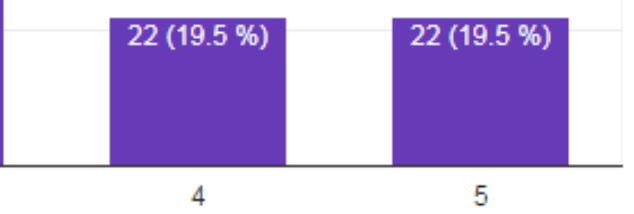

Fuente: Elaboración propia con información de la encuesta.

Ahora bien, el significado de ciudadanía según los estudiantes universitarios encuestados, principalmente es tener derechos y obligaciones con un $73.5 \%$ de las respuestas, pertenecer a un país con un $10.6 \%$ y tener responsabilidades con un $8.8 \%$ de las menciones establecidas en la gráfica 4.

Gráfica 4. Significado de ciudadanía según los estudiantes universitarios.
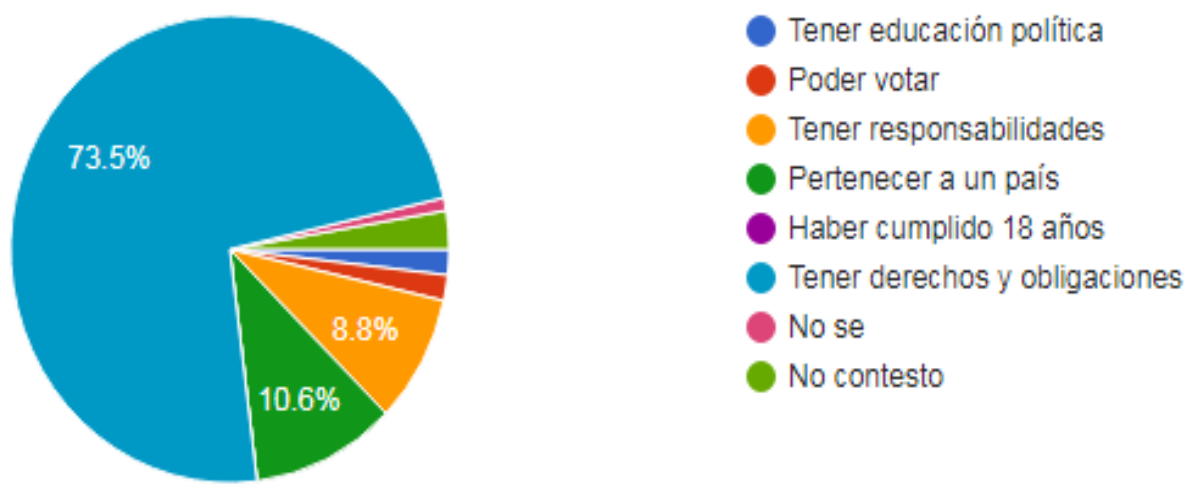

Fuente: Elaboración propia con información de la encuesta. 
Por último, la percepción de los estudiantes respecto al grado en que los ciudadanos pueden influir en las decisiones de un gobierno, (Gráfica 5); se registró en una escala del 1 al 5; siendo 1 mucho y 5 nada; concentrándose las opiniones en la escala 1 mucho con $28.3 \%$, seguido de la escala 3 con $24.8 \%$ y luego de la escala 4 con un $20.4 \%$. En resumen, lo anterior significa que $45.1 \%$ afirman que los ciudadanos pueden influir mucho, $24.8 \%$ que poco, y $30.1 \%$ que nada.

Gráfica 5. Percepción de los estudiantes respecto a la influencia que los ciudadanos pueden ejercer en decisiones de gobierno.

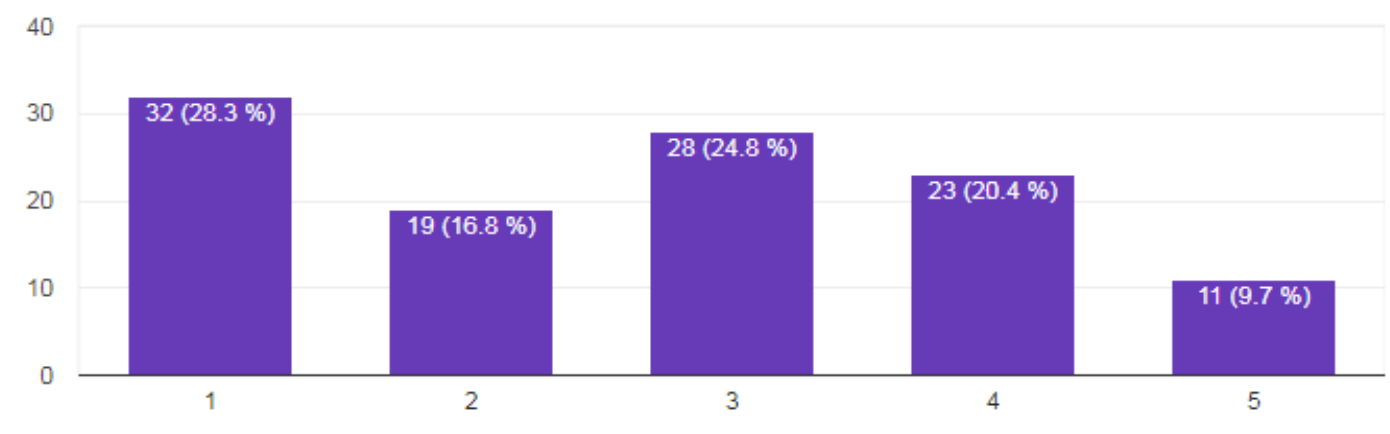

Fuente: Elaboración propia con información de la encuesta.

Por todos los resultados presentados, se evidencia que la formación cívica permite el desarrollo de una ciudadanía informada, responsable que participa activamente y que percibe la democracia como factor indispensable para der legitimidad a un gobierno.

Sin embargo, se debe aceptar que en México se adolece de continuidad en la construcción de una cultura cívica que inculque a los ciudadanos desde pequeños, los valores de tolerancia y respeto por los demás; y que también permita edificar un criterio de ciudadano global capaz de observar su entorno y en afán de mejorar, proponer cambios, participar proactivamente, asumir actitudes y manifestar conductas que muestren su valor cívico y ético en su diario vivir. Por tanto, se tiene que recorrer un largo camino para aspirar a formar ciudadanos informados, responsables, que participan activamente en su entorno social. 


\section{Formación cívica, estructura fundamental de la participación ciudadana}

Volumen 12, Número 22 ene-jun 2020

Monterde Valenzuela María de los Ángeles y

Morales Tostado María del Carmen

Al revisar los antecedentes y evolución de las políticas públicas de educación cívica en México, se observa un impulso sostenido en la formación de valores como patriotismo, democracia, participación, entre otros, en nivel de educación básico; y en media superior y superior el esfuerzo es inconsistente y devela falta de estrategia que permita estimar este impacto en la formación de las generaciones de ciudadanos. Al respecto la propuesta es diseñar una estrategia incluyente (todos los sectores); continua (a pesar de los sexenios) y práctica (con acciones de valor cívico y ético).

Se considera oportuno proteger lo básico y elemental de la temática que favorece la formación cívica y que si se presenta la necesidad de cambiar los modelos educativos se procure que la transición de un modelo a otro permitiendo la actualización y continuidad en los temas centrales de formación cívica y ética, en su comprensión y práctica.

Así mismo, es importante en la puesta en práctica de las estrategias de políticas educativas sobre cultura cívica, se cuente con docentes mejor capacitados ya que de ello dependerá el éxito en el aprendizaje de los estudiantes.

Se destaca la participación femenina en esta encuesta, correspondiendo al $63.7 \%$ de mujeres. Impactante resultó el 99\% de los estudiantes que coinciden en que los problemas de la sociedad deben ser resueltos por la sociedad y el gobierno; pero contradice lo expresado anteriormente, ya que como ciudadano no se asume como actor activo del entorno democrático.

La televisión, fue el medio de pasatiempo muy utilizado y contribuyó notablemente en la adquisición de nuevos hábitos de consumo, utilización del tiempo, cambios en las dinámicas familiares y formación de la conciencia individual y colectiva. Hoy los medios digitales ocupan este privilegiado lugar.

Los universitarios expresan que el principal lugar de reflexión sobre temas políticos en México es la escuela, después la familia y luego el grupo de amigos; y que las fuentes primordiales de información utilizadas para conocer el entorno político de México, son las redes sociales y la televisión, situación que devela un gran reto para las instituciones educativas en el sentido de formar ciudadanía.

Preocupante resultó conocer que el $69.9 \%$ de los encuestados, considera "en parte" que México es un país democrático; infiriendo de esta percepción que existe poco convencimiento de la respuesta otorgada o tener alta expectativa de lo que es democracia. 
Cierto es que los encuestados pertenecen a generaciones digitales, las cuales extraen todo su vivir en estos medios de comunicación, resultando asombroso percatarse como se manejan, exploran y confían en la información proveniente de ellos. Por tal motivo, es ineludible proponer una formación cívica que le permita conocer, discernir, analizar y tomar decisiones ciudadanas. Además, y derivado de este punto, es necesario cuidar la ética en gestión de la información.

Es determinante la función de las universidades públicas y privadas en la formación del talento; la capacidad de análisis, el manejo del debate de forma pacífica, y la presentación de propuestas como parte de las actividades diseñadas desde la academia para incentivar la participación ciudadana.

\section{REFERENCIAS BIBLIOGRÁFICAS}

COMISIÓN ECONÓMICA PARA AMÉRICA LATINa. (03 de 2011). Cepal.Org. Obtenido de https://biblioguias.cepal.org/c.php?g=159515\&p=1044440

CONDE , S., Formación Ciudadana en México, en Cuadernos de Divulgación de la Cultura Democrática \#32, méxico, INE,2016,p.1 [en línea]Disponible en:

https://portalanterior.ine.mx/archivos2/portal/historico/contenido/recursos/IFEv2/DECEYEC/DECEYEC-CuadernosdeDivulgacion/docs/32.pdf

GOBIERNO DE MÉXICO. (2013). pnd.gob.mx [en línea]Disponible en: http://pnd.gob.mx/wp-content/uploads/2013/05/PND.pdf

GONZÁLEZ LUNA corvera, T. (2016). INE, Democracia y Formación de Ciudadanía. Cuaderno de divulgación de la cultura democrática \#28. [en línea]Disponible en: https://portalanterior.ine.mx/archivos2/portal/historico/contenido/recursos/IFEv2/DECEYEC/DECEYEC-CuadernosdeDivulgacion/docs/28.pdf

GONZÁLEZ, A. Plan-nacional-de-desarrollo-2008-2014.México, 2019 [en línea]Disponibe en: https://newsweekespanol.com/2019/05/opinion-plan-nacional-de-desarrollo-fin-deloscurantismo-neoliberal

GUERRERO Useda, M. E., \& Gómez Paternina, D. A. Enseñanza de la ética y la educación moral, ¿permanecen ausentes de los programas universitarios? Revista electrónica de investigación educativa,2013.

GUEVARA Niebla,G., Democracia y Educación en Cuaderno de Divulgación de la Cultura Democrática \#16,México,INE,2016, p.77[en línea] Disponible en: https://portalanterior.ine.mx/archivos2/portal/historico/contenido/recursos/IFEv2/DECEYEC/DECEYEC-CuadernosdeDivulgacion/docs/16.pdf

LATAPÍ SARRe, P., El Debate sobre los valores en la Escuela Mexican,México, Fondo de Cultura Económica,2013. 


\section{Formación cívica, estructura fundamental de la participación ciudadana}

Merino, M. (2016). INE. La participación Ciudadana en la Democracia. Cuaderno de divulgación de la Cultura Democrática \#4 [en línea]Disponible en: https://portalanterior.ine.mx/archivos2/portal/historico/contenido/recursos/IFEv2/DECEYEC/DECEYEC-CuadernosdeDivulgacion/docs/04.pdf

Morales Tostado, M., \& Monterde Valenzuela, M. (15 de 09 de 2018). Formulario de Google "Cultura Cívica Democrática". Hermosillo, Sonora, México.

OCDE. (2015). Gobierno Abierto en América Latina. Estudios sobre Gobernanza Pública, España, OCDE Publishing,2015,p.s/n [ en línea] Disponible en: http://www.oecd.org/gov/gobierno-abierto-en-america-latina-9789264225787-es.htm

OEA Consejo Permanente. Oas.org/Carta Democrática Interamericana [en línea]Disponible en: http://www.oas.org/OASpage/esp/Publicaciones/CartaDemocratica_spa.pdf,2003

RAMÍREZ,Gloria, Coord.,Plan Nacional de educación en Derechos humanos, Primera edición,México 2006,Cátedra UNESCO de Derechos Humanos de la UNAM Facultad de Ciencias Políticas y Sociales (FCPS)

SESENTO GARCÍA, L., La influencia de los medios de comunicación en las contribuciones a las ciencias sociales, Eumed,2015

SOJO-GARZA ALDAPE, E., De la alternancia al desarrollo: Políticas públicas del gobierno del cambio, México,Fondo de Cultura Económica,2005

\section{CUADROS Y GRÁFICAS}

Cuadro 1. Datos generales de la población universitaria objeto de estudio.

Gráfica 1. Lugar donde se reflexiona sobre temas políticos en México

Gráfica 2. Percepción de la democracia en México según estudiantes universitarios

Gráfica 3. Escala de satisfacción con la democracia en México

Gráfica 4. Significado de ciudadanía según los estudiantes

Gráfica 5. Percepción de los estudiantes respecto a la influencia que los ciudadanos pueden ejercer en las decisiones de gobierno.

Recibido 12 dejunio de 2020

Aceptado 30 de Junio de 2020 